\title{
ELECTROMAGNETIC DOSIMETRY BASED ON EFIE FORMULATION AND RWG BASIS FUNCTION
}

\author{
MARIO CVETKOVIĆ \& DRAGAN POLJAK \\ University of Split, Croatia
}

\begin{abstract}
The paper deals with the electromagnetic dosimetry model for the lossy homogeneous biological body. The model is based on the use of electric field integral equation (EFIE) in the frequency domain derived using the surface equivalence theorem and the boundary conditions for the electric field. The scattered electric field is expressed in terms of the equivalent electric and magnetic current densities which are expanded using Rao-Wilton-Glisson (RWG) basis function, and a point wise orthogonal $\hat{n} \times \mathrm{RWG}$ basis function, respectively. Some illustrative results for the obtained equivalent surface current densities are given. The electromagnetic model can be useful in high frequency dosimetry of the human brain and also in modeling of medical technique such as transcranial magnetic stimulation (TMS).

Keywords: electric field integral equation, surface integral equation, equivalence principle, $R W G$ basis functions, human exposure to electromagnetic fields, computational electromagnetics.
\end{abstract}

\section{INTRODUCTION}

In addition to the environmental exposure to artificially generated electromagnetic (EM) fields due to increasing number of power and communication equipment, the efficient applications of EM radiation in medical treatments and diagnosis also require the knowledge of the accurate distribution of these fields inside the biological tissues. As the direct measurement of these quantities is rather difficult, or even impossible, the computational methods have become necessary to determine the internal field distributions [1]-[4].

From the first days, the computational bioelectromagnetics models formulated using the differential equation approach became the standard, although the integral equation approach, using the Green integral representation, is more suitable in case of open boundary problems such as the human body exposed to incident EM field [5], [3]. Although the numerical methods based on the solution of integral equations in computational electromagnetics (CEM) were developed more than half a century ago [6], [7], only recently have they begun to attract more attention in the bioelectromagnetics community as seen from a number of published papers [2], [3], [5], [8], [9].

In order to formulate the bioelectromagnetics problem using the integral equation approach, one can carry out similar procedure as in a case of classical scattering problem [5]. In case of the homogeneous biological body of an arbitrary shape $S$ representing the scatterer, placed in the incident electromagnetic field, the electric field integral equation (EFIE), based on the application of surface equivalence theorem can be used.

This paper features the application of EFIE to the problem of a biological body, i.e. the human brain exposed to high frequency electromagnetic radiation and also to the field due to a TMS stimulation coil.

The paper is organized as follows: following the introductory part, in the second part the surface integral equation set is derived using the surface equivalence theorem and the boundary conditions for the electric field. Some details on the expansion of the introduced equivalent surface current densities using the so called Rao-Wilton-Glisson (RWG) basis function are also given. This is followed by some numerical results for the equivalent current densities and the comparison with the commercial software. Furthermore, some 

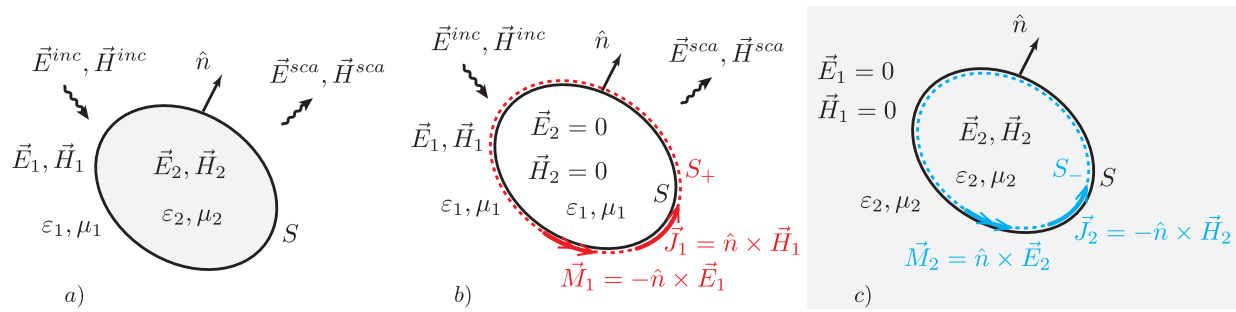

Figure 1: Biological body as a lossy homogeneous dielectric $\left(\varepsilon_{2}, \mu_{2}\right)$ placed in the incident field $\left(\vec{E}^{i n c}, \vec{H}^{i n c}\right)$. (a) Original problem; (b) Equivalent problem for the outside; (c) Equivalent problem for the inside.

illustrative results are shown related to application of our model formulated using EFIE to the bioelectromagnetics problem. Finally, some concluding remarks are given.

\section{THEORETICAL BACKGROUND}

\subsection{Surface integral equation formulation}

The electromagnetic wave incident on the homogeneous biological body can be treated as a classical scattering problem. Applying the surface equivalence theorem and the boundary conditions for the electric field one can formulate the electromagnetic model as the surface integral equation (SIE), i.e. the electric field integral equation (EFIE).

To apply the equivalence theorem, the biological body is first replaced by a homogeneous dielectric body of arbitrary shape $S$ placed in a free space with a given properties $\left(\varepsilon_{1}, \mu_{1}\right)$, as shown in Fig. 1(a). The properties of the biological object are represented by complex permitivity and permeability $\left(\varepsilon_{2}, \mu_{2}\right)$, where the complex permitivity is given by

$$
\varepsilon_{2}=\varepsilon_{0} \varepsilon_{r}-j \frac{\sigma}{\omega}
$$

where $\varepsilon_{0}$ is permitivity of the free space, $\varepsilon_{r}$ is relative permitivity, $\sigma$ is electrical conductivity of the biological body, and $\omega=2 \pi f$ is the operating frequency. The value for the permeability can be taken as $\mu_{0}=4 \pi \times 10^{-7} \mathrm{Vs} / \mathrm{Am}$, as the biological tissues do not posses magnetic properties.

The lossy homogeneous object representing, e.g. the brain, is exposed to radiation of a known electromagnetic (EM) field $\left(\vec{E}^{i n c}, \vec{H}^{i n c}\right)$. The incident EM field is present regardless of the presence of the material body. As the scattering object is present, there will also be the scattered field, denoted by $\left(\vec{E}^{s c a}, \vec{H}^{s c a}\right)$. The fields exterior and interior to the surface $S$ are, $\left(\vec{E}_{1}, \vec{H}_{1}\right)$ and $\left(\vec{E}_{2}, \vec{H}_{2}\right)$, respectively. Also, in Fig. 1, unit vector $\hat{n}$ placed on the surface $S$ pointing from the region 2 into region 1 , can be noticed.

Using the equivalence theorem for the surface $S$ of the biological body, two equivalent problems can be formulated, in terms of the equivalent electric and magnetic current densities $\vec{J}$ i $\vec{M}$ placed on the surface $S$, one for the region 1 (outside) and other for the region 2 (inside) [10], [7], [6], [11]. The two equivalent problems are shown on Figs 1(b) and 1(c).

In the case of an external equivalent problem, shown in Fig. 1(b), field inside is assumed to be zero, $\left(\vec{E}_{2}=0, \vec{H}_{2}=0\right)$, hence allowing one to replace material properties of the region. Selecting the same properties as that of an exterior region, leads to the homogeneous domain 
of $\left(\varepsilon_{1}, \mu_{1}\right)$, allowing one to use the free space Green's function. The boundary conditions on the surface $S$ are satisfied by introducing equivalent surface currents $\vec{J}_{1}$ and $\vec{M}_{1}$ at the surface $S$. Following the same procedure for the interior equivalent problem, yields another homogeneous domain of $\left(\varepsilon_{2}, \mu_{2}\right)$. Again, the equivalent surface currents $\overrightarrow{J_{2}}=-\overrightarrow{J_{1}}$ and $\vec{M}_{2}=-\vec{M}_{1}$ are introduced, as indicated in Fig. 1(c).

As both problems represent equivalent current densities radiating in a homogeneous medium, the following expressions for scattered fields can be used

$$
\begin{aligned}
& \vec{E}_{n}^{s c a}(\vec{J}, \vec{M})=-j \omega \vec{A}_{n}-\nabla \varphi_{n}-\frac{1}{\varepsilon_{n}} \nabla \times \vec{F}_{n}, \\
& \vec{H}_{n}^{s c a}(\vec{J}, \vec{M})=-j \omega \vec{F}_{n}-\nabla \psi_{n}+\frac{1}{\mu_{n}} \nabla \times \vec{A}_{n},
\end{aligned}
$$

where $n=1,2$ is index of the medium where equivalent surface currents radiate, and $\varphi, \vec{F}$, $\psi$ i $\vec{A}$ are scalar and vector, electric and magnetic potentials, respectively, given in terms of integrals over the sources, i.e.

$$
\begin{gathered}
\vec{A}_{n}(\vec{r})=\mu_{n} \iint_{S} \vec{J}\left(\vec{r}^{\prime}\right) G_{n}\left(\vec{r}, \vec{r}^{\prime}\right) \mathrm{d} S^{\prime}, \\
\vec{F}_{n}(\vec{r})=\varepsilon_{n} \iint_{S} \vec{M}\left(\vec{r}^{\prime}\right) G_{n}\left(\vec{r}, \vec{r}^{\prime}\right) \mathrm{d} S^{\prime}, \\
\varphi_{n}(\vec{r})=\frac{j}{\omega \varepsilon_{n}} \iint_{S} \nabla_{S}^{\prime} \cdot \vec{J}\left(\vec{r}^{\prime}\right) G_{n}\left(\vec{r}, \vec{r}^{\prime}\right) \mathrm{d} S^{\prime}, \\
\psi_{n}(\vec{r})=\frac{j}{\omega \mu_{n}} \iint_{S} \nabla_{S}^{\prime} \cdot \vec{M}\left(\vec{r}^{\prime}\right) G_{n}\left(\vec{r}, \vec{r}^{\prime}\right) \mathrm{d} S^{\prime},
\end{gathered}
$$

where

$$
G_{n}\left(\vec{r}, \vec{r}^{\prime}\right)=\frac{e^{-j k_{n} R}}{4 \pi R} ; \quad R=\left|\vec{r}-\vec{r}^{\prime}\right| .
$$

$G_{n}\left(\vec{r}, \vec{r}^{\prime}\right)$ is Green's function for the homogeneous medium $n, R$ is the distance from observation point $\vec{r}$ to source point $\vec{r}^{\prime}$, while $k_{n}$ is wave number in medium $n,(n=1,2)$.

The electric and magnetic charges from (6) and (7), respectively, can be replaced with the divergence of the electric and magnetic currents, respectively, if the continuity equation is used.

Using (4)-(7), the scattered field from (2) and (3) can be expressed in terms of the equivalent surface currents. Applying the boundary conditions at the surface $S$ being interface of the two equivalent problems, a set of four equations for electric and magnetic field, respectively, is obtained. Choosing the two for the electric field, yields the electric field integral equation (EFIE) formulation in the frequency domain for the lossy homogeneous biological object

$$
\left[-\vec{E}_{n}^{s c a}(\vec{J}, \vec{M})\right]_{t a n}= \begin{cases}{\left[\vec{E}^{i n c}\right]_{t a n},} & n=1, \\ 0, & n=2,\end{cases}
$$

where $\vec{E}^{i n c}$ is the known incident field, while $\vec{J}$ and $\vec{M}$ represent the unknown surface currents. 
Inserting (4)-(7) into (2) and (3) we arrive at the coupled set of integral equations

$$
\begin{aligned}
& j \omega \mu_{n} \iint_{S} \vec{J}\left(\vec{r}^{\prime}\right) G_{n}\left(\vec{r}, \vec{r}^{\prime}\right) \mathrm{d} S^{\prime}-\frac{j}{\omega \varepsilon_{n}} \nabla \iint_{S} \nabla_{S}^{\prime} \cdot \vec{J}\left(\vec{r}^{\prime}\right) G_{n}\left(\vec{r}, \vec{r}^{\prime}\right) \mathrm{d} S^{\prime}+ \\
& +\nabla \times \iint_{S} \vec{M}\left(\vec{r}^{\prime}\right) G_{n}\left(\vec{r}, \vec{r}^{\prime}\right) \mathrm{d} S^{\prime}= \begin{cases}\vec{E}^{i n c}, & n=1, \\
0, & n=2,\end{cases}
\end{aligned}
$$

where $\vec{J}$ and $\vec{M}$ are unknown equivalent surface electric and magnetic currents.

After some mathematical manipulations, details of which can be found in [8], the gradient operator outside the second integral of (10) and curl operator outside the third integral can be transferred to the Green's function

$$
\begin{aligned}
& j \omega \mu_{n} \iint_{S} \vec{J}\left(\vec{r}^{\prime}\right) G_{n}\left(\vec{r}, \vec{r}^{\prime}\right) \mathrm{d} S^{\prime}-\frac{j}{\omega \varepsilon_{n}} \iint_{S} \nabla_{S}^{\prime} \cdot \vec{J}\left(\vec{r}^{\prime}\right) \nabla G_{n}\left(\vec{r}, \vec{r}^{\prime}\right) \mathrm{d} S^{\prime}+ \\
& +\iint_{S} \vec{M}\left(\vec{r}^{\prime}\right) \times \nabla^{\prime} G_{n}\left(\vec{r}, \vec{r}^{\prime}\right) \mathrm{d} S^{\prime}= \begin{cases}\vec{E}^{i n c}, & n=1, \\
0, & n=2,\end{cases}
\end{aligned}
$$

where the following property for the Green's function gradient has been used

$$
\nabla G_{n}\left(\vec{r}, \vec{r}^{\prime}\right)=-\nabla^{\prime} G_{n}\left(\vec{r}, \vec{r}^{\prime}\right) .
$$

\subsection{Expansion of surface currents}

In the case of a complex surface geometry, such as e.g. human brain, the coupled integral equations set (11) cannot be solved analytically, hence the numerical solution is necessary.

An efficient scheme of a method of moments (MoM) is implemented in this work. First, the equivalent electric and magnetic currents $\vec{J}$ and $\vec{M}$ in (11) are expanded in terms of a linear combination of known basis functions $\vec{f}_{n}$ and $\vec{g}_{n}$, respectively

$$
\begin{aligned}
\vec{J}(\vec{r}) & =\sum_{n=1}^{N} J_{n} \vec{f}_{n}(\vec{r}), \\
\vec{M}(\vec{r}) & =\sum_{n=1}^{N} M_{n} \vec{g}_{n}(\vec{r}),
\end{aligned}
$$

where $J_{n}$ and $M_{n}$ are unknown coefficients, while $N$ stands for total number of elements used to discretize the surface $S$.

If the surface of a biological body is discretized using the triangular elements, the so called Rao-Wilton-Glisson (RWG) basis functions [12] specially developed for triangular patches can be used.

RWG basis function $\vec{f}_{n}$, or, as sometimes called the edge-element, is defined on $T_{n}^{+}$and $T_{n}^{-}$pair of triangles sharing a common edge, as shown on Fig. 2.

Namely, the function is given by

$$
\vec{f}_{n}^{ \pm}(\vec{r})= \begin{cases}\frac{l_{n}}{2 A_{n}^{ \pm}} \vec{\rho}_{n}^{ \pm}, & \vec{r} \in T_{n}^{ \pm}, \\ 0, & \vec{r} \notin T_{n}^{ \pm},\end{cases}
$$




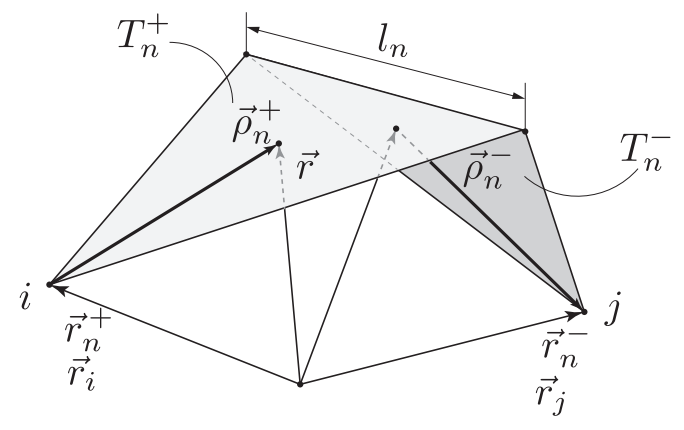

Figure 2: RWG basis function $\vec{f}_{n}(\vec{r})$ defined on a pair of triangles in $\mathbf{R}^{3}$ [12].

where $l_{n}$ is the edge length at the interface between triangles $T_{n}^{+}$and $T_{n}^{-}$, while $A_{n}^{+}$and $A_{n}^{-}$denote the surface areas of those triangles. The vector $\vec{\rho}_{n}^{+}=\vec{r}-\vec{r}_{n}^{+}$is directed from the free vertex of $T_{n}^{+}$and $\vec{\rho}_{n}^{-}=\vec{r}_{n}^{-}-\vec{r}$ is directed to the free vertex of $T_{n}^{-}$, as shown on Fig. 2.

While the surface electric current $\vec{J}$ is approximated by the RWG function $\vec{f}_{n}$, the surface magnetic current $\vec{M}$ is expanded by $\vec{g}_{n}=\hat{n} \times \vec{f}_{n}$, i.e. the function point wise orthogonal to the RWG function. Fig. 3 depicts the linear combination of a three basis functions (showed only on one triangle) obtained using the RWG and $\hat{n} \times \mathrm{RWG}$ function, respectively.
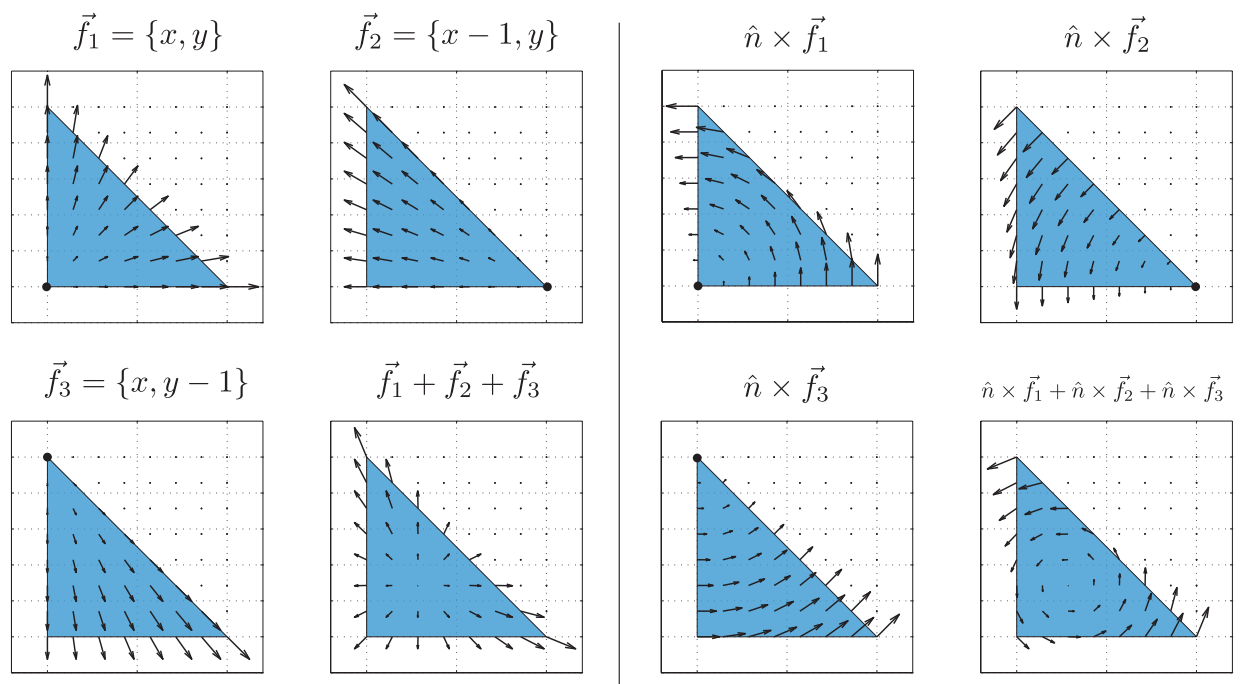

a)
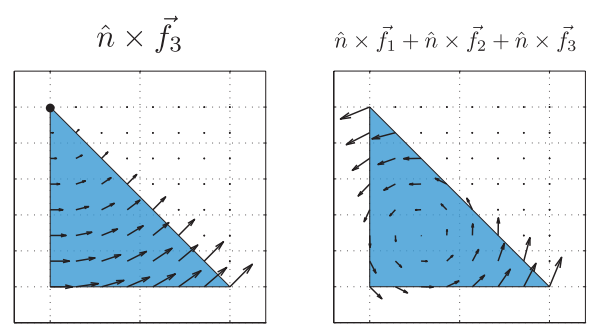

b)

Figure 3: Three basis functions associated with three sides (edges) of a triangle and their linear combination, using: (a) RWG function; (b) $\hat{n} \times$ RWG function. 
The unknown equivalent currents $\vec{J}\left(\vec{r}^{\prime}\right)$ and $\vec{M}\left(\vec{r}^{\prime}\right)$ from (11) are substituted by (13) and (14). Eqn (11) is next multiplied by the set of a test functions $\vec{f}_{m}$, where $\vec{f}_{m}=\vec{f}_{n}$, and integrated over the surface $S$.

Undertaking some additional mathematical manipulations [5], [8], yields

$$
\begin{gathered}
j \omega \mu_{i} \sum_{n=1}^{N} J_{n} \iint_{S} \vec{f}_{m}(\vec{r}) \cdot \iint_{S^{\prime}} \vec{f}_{n}\left(\vec{r}^{\prime}\right) G_{i} d S^{\prime} d S+ \\
+\frac{j}{\omega \varepsilon_{i}} \sum_{n=1}^{N} J_{n} \iint_{S} \nabla_{S} \cdot \vec{f}_{m}(\vec{r}) \iint_{S^{\prime}} \nabla_{S}^{\prime} \cdot \vec{f}_{n}\left(\vec{r}^{\prime}\right) G_{i} d S^{\prime} d S+ \\
\pm \sum_{n=1}^{N} M_{n} \iint_{S} \vec{f}_{m}(\vec{r}) \cdot\left[\hat{n} \times \vec{g}_{n}\left(\vec{r}^{\prime}\right)\right] d S+ \\
+\sum_{n=1}^{N} M_{n} \iint_{S} \vec{f}_{m}(\vec{r}) \cdot \iint_{S^{\prime}} \vec{g}_{n}\left(\vec{r}^{\prime}\right) \times \nabla^{\prime} G_{i} d S^{\prime} d S= \\
= \begin{cases}\iint_{S} \vec{f}_{m}(\vec{r}) \cdot \vec{E}^{i n c} d S, & i=1 \\
0, & i=2,\end{cases}
\end{gathered}
$$

where subscript $i$ stands for the region index. After extracting the two sums, (16) can be written as the following system of linear equations

$$
\sum_{n=1}^{N}\left(j \omega \mu_{i} A_{m n, i}+\frac{j}{\omega \varepsilon_{i}} B_{m n, i}\right) J_{n}+\sum_{n=1}^{N}\left(C_{m n, i}+D_{m n, i}\right) M_{n}= \begin{cases}V_{m}, & i=1 \\ 0, & i=2\end{cases}
$$

Recasting the expression (17) in the matrix form, leads to

$$
[\mathbf{Z}] \cdot\{\mathbf{I}\}=\{\mathbf{V}\},
$$

where $\mathbf{Z}$ is the $2 N \times 2 N$ matrix of the system, while $\mathbf{V}$ represents the source vector of a dimension $2 N$.

Solving (18) results in vector I containing the unknown coefficients $J_{n}$ and $M_{n}$. From these coefficients, using (13) and (14), the equivalent electric and magnetic currents, $\vec{J}$ and $\vec{M}$, respectively, could be determined.

From the equivalent surface currents, the electric field inside the biological body can be determined using the following:

$$
\begin{aligned}
\vec{E}_{2}(\vec{r})=-j \omega \mu_{2} \iint_{S} \vec{J}\left(\vec{r}^{\prime}\right) G_{2}\left(\vec{r}, \vec{r}^{\prime}\right) d S^{\prime}-\frac{j}{\omega \varepsilon_{2}} & \iint_{S} \nabla_{S}^{\prime} \cdot \vec{J}\left(\vec{r}^{\prime}\right) G_{2}\left(\vec{r}, \vec{r}^{\prime}\right) d S^{\prime}- \\
& -\iint_{S} \vec{M}\left(\vec{r}^{\prime}\right) \times \nabla G_{2}\left(\vec{r}, \vec{r}^{\prime}\right) d S^{\prime} .
\end{aligned}
$$

\section{NUMERICAL RESULTS}

The results obtained using the SIE formulation are compared to the results from the commercially available software FEKO. The first computational example is related to the dielectric sphere of a radius $r=3 \mathrm{~cm}$, with parameters $\varepsilon_{r}=35$ and $\sigma=0.7 \mathrm{~S} / \mathrm{m}$, placed in the field of a $918 \mathrm{MHz}$ plane wave, directed in the negative $z$ coordinate, polarized in $x$ 
direction, as shown on Fig. 4. The second example is the dielectric cube with side $a=1 \mathrm{~m}$, $k a=1$, frequency of incident wave $f=47.74 \mathrm{MHz}, \varepsilon_{r}=54.5$, and $\sigma=1.35 \mathrm{~S} / \mathrm{m}$.

The incident electric field value in both cases is $E_{0}^{i n c}=86.83 \mathrm{~V} / \mathrm{m}$, corresponding to power density of $P_{0}^{i n c}=1 \mathrm{~mW} / \mathrm{cm}^{2}$. The surface of the sphere is discretized by $T=152$ triangles, corresponding to $N=228$ edge-elements, while the cube surface is discretized using $T=768$ triangles, corresponding to $N=1152$ edge-elements.

The distribution of the equivalent surface electric and magnetic currents obtained by the proposed model and using the FEKO are shown in Figs 4(a)-(b) and 4(c)-(d), respectively, while the maximum values obtained for $\mathbf{J}_{\max }$ and $\mathbf{M}_{\max }$ are also compared in Table 1.

It can be clearly seen that the maximum values for the equivalent surface currents obtained using EFIE formulation is similar to the results given by commercial software. Moreover, the obtained distribution of these equivalent surface currents are rather comparable.

As seen from Fig. 4, a much coarser mesh, compared to FEKO, has been used in the proposed method, in order to facilitate the solution process. Although the proposed method is applicable to an arbitrarily shaped biological object with more detailed geometry, the present implementation is limited to a roughly several thousand elements. The higher number of elements would consequently lead to a very large matrix system whose solution would

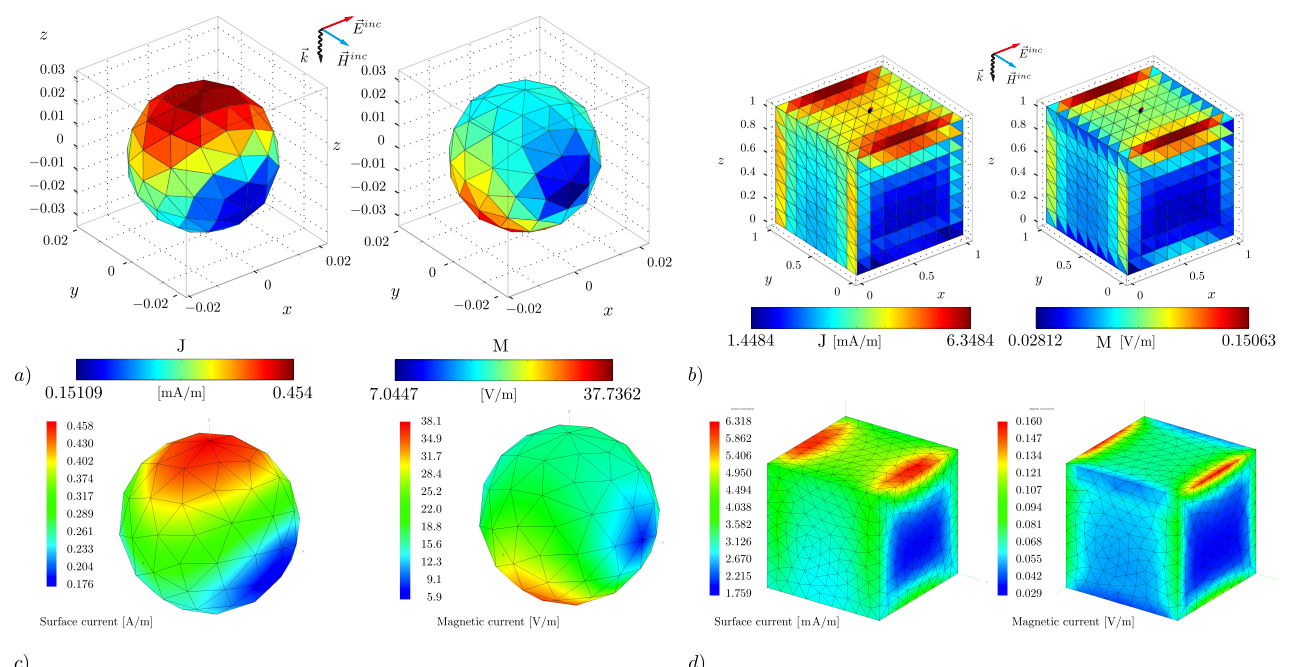

c)

Figure 4: Distribution of the equivalent electric current $\vec{J}$ and magnetic current $\vec{M}$, respectively, on the surface $S$ of a sphere and a unit cube due to vertically incident, horizontally polarized plane wave. Results obtained using EFIE formulation: (a) and (b). Results obtained using FEKO: (c) and (d).

Table 1: Comparison of maximum values for the surface electric and magnetic currents obtained by EFIE formulation and commercial software.

\begin{tabular}{l|ll|ll}
\hline & Sphere & & Cube \\
\hline & EFIE & FEKO & EFIE & FEKO \\
\hline$J_{\max }[\mathrm{mA} / \mathrm{m}]$ & 4.0209 & 3.947 & 6.348 & 6.318 \\
$M_{\max }[\mathrm{V} / \mathrm{m}]$ & 0.8706 & 0.843 & 0.151 & 0.160 \\
\hline
\end{tabular}



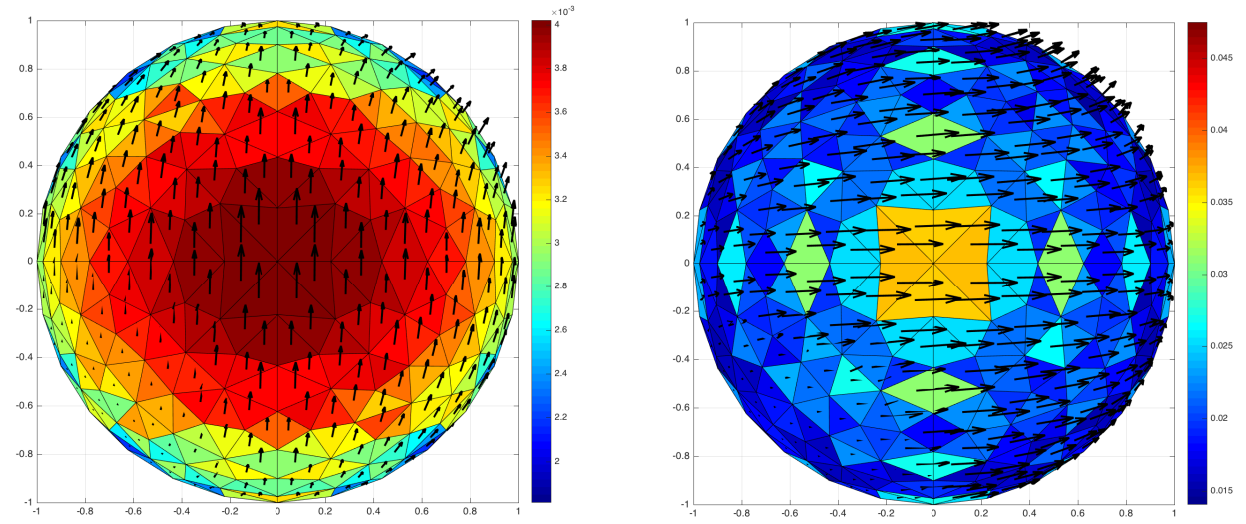

Figure 5: Distribution of the equivalent currents on the surface of a sphere due to vertically incident plane wave of frequency $f=300 \mathrm{MHz}$. Results for the equivalent electric current $\vec{J}$ (left), and the equivalent magnetic current $\vec{M}$ (right).

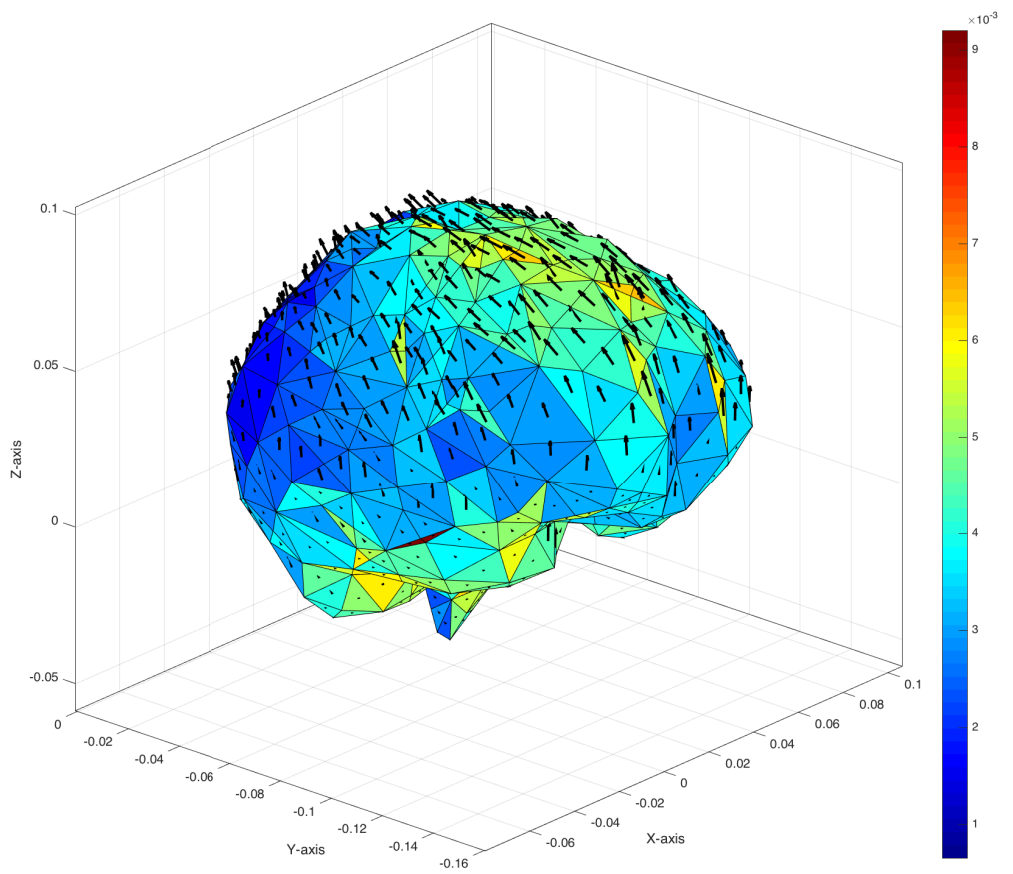

Figure 6: Distribution of the equivalent electric currents on the surface of a human brain model to vertically incident plane wave of frequency $f=900 \mathrm{MHz}$.

thus require the use of advanced techniques such as multilevel fast multipole method. The comparison of the proposed method to other numerical methods in case of low frequency field exposure could be found in [4]. 


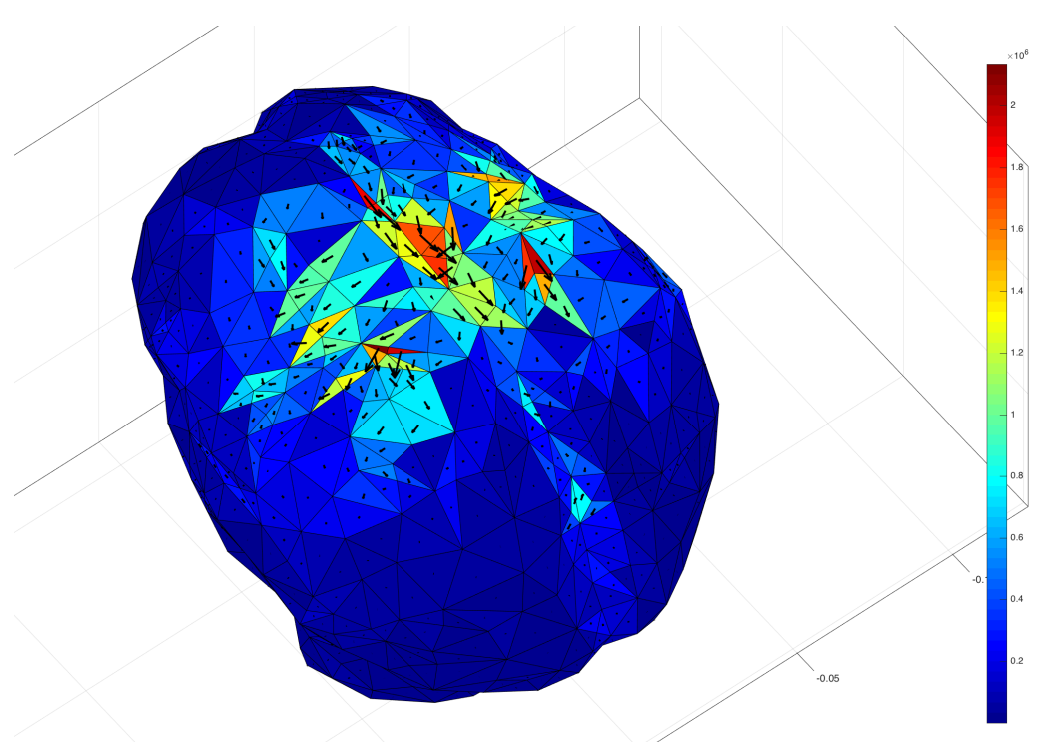

Figure 7: Distribution of the equivalent electric currents on the surface of a human brain model due to a transcranial magnetic stimulation coil positioned $1 \mathrm{~cm}$ over the brain surface.

Additionally, only as an illustrative results, Fig. 5 shows the distribution of the equivalent electric and magnetic currents, respectively, on a more finely discretized spherical surface due to incident wave of $300 \mathrm{MHz}$. One can note on each triangular element the electric current vector (expanded using RWG function) being piecewise orthogonal to the corresponding magnetic current vector (expanded using $\hat{n} \times \mathrm{RWG}$ function).

Finally, some numerical results, related to the homogeneous human brain exposed to EM radiation, are given. The results for the equivalent surface electric current, obtained using the EFIE formulation (10), and the RWG basis functions (15), are shown on Figs 6 and 7.

Fig. 6, depicting the human brain exposed to electromagnetic wave incident from the above, corresponds to high frequency exposure scenario [13], while Fig. 7 is related to the human brain stimulated by a transcranial magnetic stimulation (TMS) figure-of-eight coil positioned $1 \mathrm{~cm}$ over the primary motor cortex [8]. As seen from 7, the distribution of the electric current densities over the brain surface is actually following the pattern similar to a so called figure-of-eight TMS coil, used as a low frequency EM source $(2.4 \mathrm{kHz})$ in this case.

\section{CONCLUSION}

The paper presented the integral equation based alternative to the nowadays most frequently used differential equation based formulation of the bioelectromagnetics problem. The electromagnetic model for the homogeneous biological body of arbitrary shape, represents a powerful, accurate and efficient alternative for the treatment of open boundary type problems such as a human body exposed to electromagnetic radiation. The unknown equivalent surface electric and magnetic current densities, respectively, are expanded using Rao-Wilton-Glisson basis functions and the point wise orthogonal function set defined on the triangular elements. The numerical solution of the electric field integral equation is carried out using the method of moments technique. Some illustrative results for the equivalent current densities on the surface of the brain model are given. The proposed method could be found useful in the 
initial assessment of the human brain exposed to high frequency EM radiation, and also in modeling medical techniques such as TMS. The future work will be related to a more realistic scenarios featuring the use of a non-homogeneous models, and the corresponding integral equation based formulations.

\section{REFERENCES}

[1] Hand, J., Modelling the interaction of electromagnetic fields (10 MHz-10 GHz) with the human body: methods and applications. Physics in Medicine and Biology, 53(16), p. R243, 2008.

[2] Poljak, D., Cavka, D., Dodig, H., Peratta, C. \& Peratta, A., On the use of the boundary element analysis in bioelectromagnetics. Engineering Analysis with Boundary Elements, 49, pp. 2-14, 2014.

[3] Poljak, D., Cvetković, M., Peratta, A., Peratta, C., Dodig, H. \& Hirata, A., On some integral approaches in electromagnetic dosimetry. BIOEM 2016, 2016.

[4] Poljak, D., Cvetković, M., Bottauscio, O., Hirata, A., Laakso, I., Neufeld, E., Reboux, S., Warren, C., Giannopoulos, A. \& Costen, F., On the use of conformal models and methods in dosimetry for nonuniform field exposure. IEEE Transactions on Electromagnetic Compatibility, 60(2), pp. 328-337, 2018.

[5] Cvetković, M. \& Poljak, D., An efficient integral equation based dosimetry model of the human brain. Proceedings of 2014 International Symposium on Electromagnetic Compatibility (EMC EUROPE) 2014, Gothenburg, Sweden, 1-4 September 2014, pp. 375-380, 2014.

[6] Poggio, A.J. \& Miller, E.K., Integral equation solutions of three-dimensional scattering problems. Computer Techniques for Electromagnetics, 2nd ed., ed. R. Mittra, Hemisphere Publishing Corporation, Chapter 4, pp. 159-264, 1987.

[7] Chew, W.C., Tong, M.S. \& Hu, B., Integral Equation Methods for Electromagnetic and Elastic Waves, Morgan \& Claypol Publishers, p. 261, 2009.

[8] Cvetković, M., Poljak, D. \& Haueisen, J., Analysis of transcranial magnetic stimulation based on the surface integral equation formulation. Biomedical Engineering, IEEE Transactions on, 62(6), pp. 1535-1545, 2015.

[9] Wei, F. \& Yilmaz, A., A More Scalable and Efficient Parallelization of the Adaptive Integral Method - Part II: BIOEM Application. IEEE T Antenn Propag, 62(2), pp. 727$738,2014$.

[10] Harrington, R., Boundary integral formulations for homogeneous material bodies. Journal of Electromagnetic Waves and Applications, 3(1), pp. 1-15, 1989.

[11] Umashankar, K., Taflove, A. \& Rao, S., Electromagnetic scattering by arbitrary shaped three-dimensional homogeneous lossy dielectric objects. IEEE Transactions on Antennas and Propagation, 34(6), pp. 758-766, 1986.

[12] Rao, S., Wilton, D.R. \& Glisson, A., Electromagnetic scattering by surfaces of arbitrary shape. IEEE Transactions on Antennas and Propagation, 30(3), pp. 409-418, 1982.

[13] Cvetković, M., Poljak, D. \& Hirata, A., The electromagnetic-thermal dosimetry for the homogeneous human brain model. Engineering Analysis with Boundary Elements, 63, pp. 61-73, 2016. 